\title{
Chromosome Study of Ethiopian Bat Species (Order Chiroptera)
}

\author{
Bekele Serbessa Tolera $^{1,{ }^{*} \text {, }}$ Mulugeta Tafere Woldegebriel ${ }^{2}$, Kifle Dagne Woldegebriel ${ }^{3}$ \\ ${ }^{1}$ Department of Biology, Wollega University, Nekemte, Ethiopia \\ ${ }^{2}$ Ethiopian Biodiversity Institute Bahir Dar Biodiversity Center, Bahir Dar, Ethiopia \\ ${ }^{3}$ Department of Microbial, Cellular and Molecular Biology, Addis Ababa University, Addis Ababa, Ethiopia
}

Email address:

bekelest@gmail.com (B. S. Tolera), taferemulugeta@gmail.com (M. T. Woldegebriel)

${ }^{*}$ Corresponding author

\section{To cite this article:}

Bekele Serbessa Tolera, Mulugeta Tafere Woldegebriel, Kifle Dagne Woldegebriel. Chromosome Study of Ethiopian Bat Species (Order Chiroptera). American Journal of Bioscience and Bioengineering. Vol. 9, No. 4, 2021, pp. 123-130. doi: 10.11648/j.bio.20210904.14

Received: July 15, 2021; Accepted: August 2, 2021; Published: August 26, 2021

\begin{abstract}
In this study, chromosome numbers and karyotypes of 11 bat species were analyzed. The animals were captured alive by using nets and handpicking and then chromosome preparations were made from bone marrow cells with colchicines method. Bats were collected from nine localities in Ethiopia, namely: Arbaminch, Batu/Ziway, Waliso, Fiche, Bishoftu/DebreZeit, Sof-Umar, Koka, Merehabete and Adaba. The species name and the chromosome number (2n) with their corresponding autosomal fundamental number (FN) obtained are: Hipposideros caffer $(2 \mathrm{n}=32, \mathrm{FN}=60 / 62)$ and Triaenops persicus $(2 \mathrm{n}=36$, $\mathrm{FN}=60)$ are belong to family Hipposideridae; Chaerephon pumilus $(2 \mathrm{n}=48$ and $\mathrm{FN}=54 / 56)$ with metacentric, acrocentric and acrocentric chromosomes, Chaerephon leucogaster $(2 \mathrm{n}=48, \mathrm{FN}=54)$, and Mops condylura $(2 \mathrm{n}=48, \mathrm{FN}=54)$ are members of the family Molossidae; Pipistrellus pipistrellus $(2 \mathrm{n}=36, \mathrm{FN}=52)$ with metacentric and acrocentric chromosomes, Neoromicia nanus $(2 \mathrm{n}=36, \mathrm{FN}=48)$, Miniopterus africanus $(2 \mathrm{n}=46, \mathrm{FN}=54)$ and Scotophilus dingani $(S$. viridis $) 2 \mathrm{n}=36$, $\mathrm{FN}=54$ ) with metacentric and acrocentric chromosomes are members of the family Vespertilionidae; Micropteropus pusillus $(2 \mathrm{n}=35 / 36, \mathrm{FN}=68)$ with all the chromosomes being biarmed belongs to family Pteropidae; Nycteris thebaica $(2 \mathrm{n}=42, \mathrm{FN}=$ 78/80) with 40 biarmed and two acrocentric chromosomes is member of family Nycteridae. Totally, 15 different types of chromosome number, fundamental number and morphology were identified. C. leucogaster has not been recorded in the Ethiopian bats list before. All of these species are karyologically described for the first time from Ethiopia. Some of the karyotypic findings in the present study are in agreement with previous reports from other countries, except for the lack of report on one species (C. leucogaster). In our study, the encountered problems include: lack of karyotypic literatures on Ethiopian bats and taxonomic identification. It is recommended that more karyotypic study of bat species in the country should be done using additional techniques and due attentions should be given to the conservations of this threatened groups of animals because they are declining in diversity as well as in density.
\end{abstract}

Keywords: Bat, Chiroptera, Chromosome Number, Fundamental Number, Karyotype

\section{Introduction}

Bats (Class Mammalia, order Chiroptera) are the second largest group of mammals next to rodents [10], and they comprise about $25 \%$ of the extant mammalian species [19]). Mitchell-Jones [17] estimated the global bat species to be more than 1000, and still Sotero-Caio et al. [23] claimed that they are more than 1300 species categorized under 21 extant families. Chiroptera is classified in two suborders,
Microchiroptera and Megachiroptera, with former comprising about 756 species in 16 families and the latter 166 species, all in a single family, Pteropedidae [26]. However, there is no agreement among different workers on the number of families, genera or species of bats [8]; [17]; [19], one reason being the subjectivity of the features used by different bat taxonomists that results in the variation of the number of taxa recognized by different investigators because of splitting or lumping of taxa [8].

About 200 species of bats have been recorded in Africa, in 
East Africa alone about 87, have been discovered [12]. According to Largen et al. [15] 74 species of bats have been recorded in Ethiopia, including Eritrea. Of these, five species (namely: Asselia patrizii, Myotis morrisi, Myotis scotti and Kerivoula eriophora) are endemic to Ethiopia [15]. Kruskop and Lavrenchenko [14] also reported another new endemic species, Plecotus balensis, from the Bale Mountains National Park. The 74 species of bats recorded for Ethiopia and Eritrea belong to nine families and thirty-one genera, from which about one-third of the genera belong to the family Vespertilionidae [15].

Bats play important roles in keeping the ecological balance, in seed dispersal and crosspollination of a number of plant species [27]. In addition, bats can be used as experimental animals in medical research [25] and as food in some Pacific Islands. Their guano is used as a fertilizer [18].

Chromosome data has greatly contributed to the understanding of the systematics, phylogeny and evolution of many groups of organisms, including bats. Many species of bats, especially those belonging to same genus, possess similar body structures, which has made bat classification a complicated matter for many years. Recently, based on chromosome information, it has been possible to distinguish many cryptic or sibling species of bats [5]. Hence, chromosome data can provide useful information to understand the diversity, systematics and phylogeny of bats [30]. To date, no chromosome study of Ethiopian bats has been published. This study was undertaken with the main objective of generating karyotypic information of some Ethiopian species of bats.

In this study bat species captured from several localities in Ethiopia were studied for chromosome number and morphology. Bats from five families and nine genera were included with total number of 11 species. To date, here are only scanty reports are found on the chromosomes of Ethiopian bats.

\section{Materials and Methods}

\subsection{Sites of Bat Specimens Collection}

The bat species and collection sites are given in Table 1 . The site selection was based on the early information gathered through literature and personal communications.

Table 1. 14 bat species collected from different localities in Ethiopia.

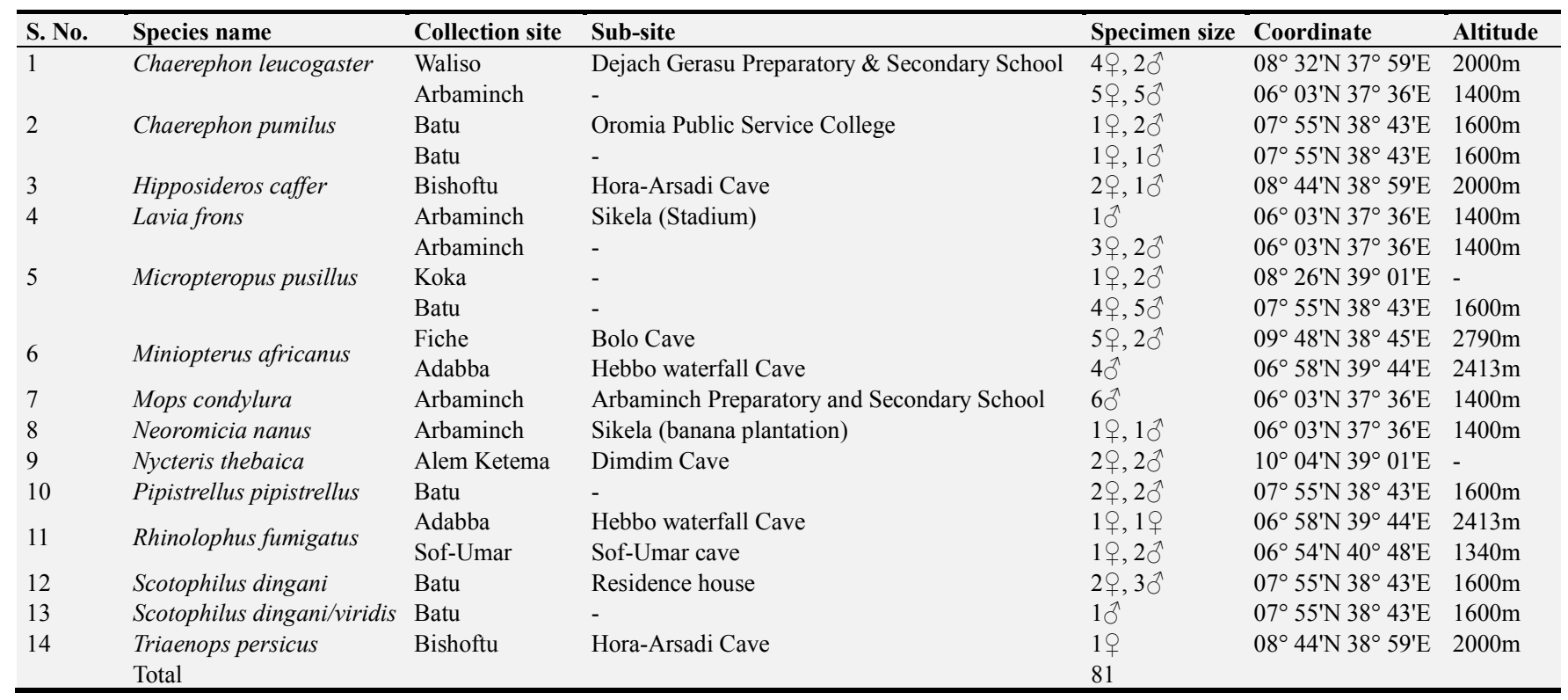

\subsection{Animal Capturing}

Bats roost in a variety of places including in caves, ceilings of buildings, crevices in tree trunks and banana leaves. Those roosting in ceilings were captured by placing a bag of fishing net at the exit point, and trapped as they are coming out for feeding around the time of sunset. Those nesting in more open places such as large caves and warehouses were captured using large insect nets. Those nesting in banana leaves were collected by hand picking wearing thick leather gloves. Captured animals were brought to a nearby school or research institute and if close to Addis
Ababa they were brought to Science Faculty for chromosome preparation and measurements of features of taxonomic importance.

\subsection{Chromosome Preparation}

Chromosome preparation was done following Lee and Elder's method cited in Hillis et al. [9] with some modifications in time of incubation and varied. Animals were injected into the peritoneal cavity with $0.1 \%$ aqueous colchicines solution at the rate of about $0.1 \mathrm{ml}$ per $10 \mathrm{gm}$ body weight. After $1 \mathrm{hr}$, the animal was sacrificed by over etherization bones of the forelimbs were dissected, crashed 
with a bone cracker in about $1 \mathrm{ml}$ of hypotonic solution $(0.075 \mathrm{M} \mathrm{KCl})$ and the bone marrow cells were allowed to swell for about half an hour at room temperature before centrifuged at $1000 \mathrm{rpm}$ for about $5 \mathrm{~min}$. The supernatant was removed and the pellet was resuspended in $1 \mathrm{ml}$ of fixative (methanol:glacial acetic acid, 3:1, v/v) and centrifuged after fixation for about $10 \mathrm{~min}$. Centrifugation and fixation were done several times. Finally, the pellet was suspended in $0.5 \mathrm{ml}$ of afresh fixative; a few drops of the suspension were splashed from a height of about half a meter on a clean glass slide, inclined at about 45 degree. The slides were allowed to air-dry at room temperature and stored away until needed for staining. Slides were stained in Giemsa stain in phosphate buffer $(\mathrm{pH}$ 6.8), rinsed in distilled water, air dried and mounted under $22 \times 55 \mathrm{~mm}$ cover slip using DPX as a mounting medium.

Metaphase cells with good chromosome spread were photographed using x100 objective of compound microscope. Karyotypes were constructed from enlarged prints of the photomicrographs. In classifying the chromosomes into groups, the Levan et al. [16] nomenclature of centromeric position was followed.

\section{Results}

For the sake of convenience karyotpe description and discussion of the results, the species were arranged according to their respective families as: Hipposideridae, Mollosidae, Nycteridae, Pteropodidae and Vespertilionidae. Here only 11 species, with good chromosome spread and clear karyotype were presented as results.

\subsection{Family Hipposideridae}

Two species, Hipposideros caffer and Triaenops persicus, were studied from the family Hiposideridae. The specimens of both species (Bish 1 \& Bish 2) were captured from Bishoftu/Debe-Zeit town while co-roosting in a cave around Hora-Arsadi Lake.

\subsubsection{Hipposideros Caffer (Sundevall, 1846)}

The karyotypes of male and female Hipposideros caffer (round-leaf bat) are presented in Figure 1A \&B. The diploid chromosome number is $2 \mathrm{n}=32$ and the autosomal fundamental number for this species is 60 . The autosomal chromosomes are metacentrics except four pair of submetacentrics. The $\mathrm{X}$ chromosome is medium sized acrocentric and $\mathrm{Y}$ is a very small acrocentric.

\subsubsection{Triaenops Persicus}

Figure 1C represents the karyotype of a female specimen of Triaenops persicus. The diploid chromosome number is $2 \mathrm{n}$ $=36$, consisting of fourteen pairs of meta-submetacentrics and four pairs of acrocentrics. The exact autosomal fundamental number cannot be determined since male karyotype is not available. However, it would be 60 or 62 , depending on whether $\mathrm{X}$ is meta-submetacentrics or acrocentrics, respectively.

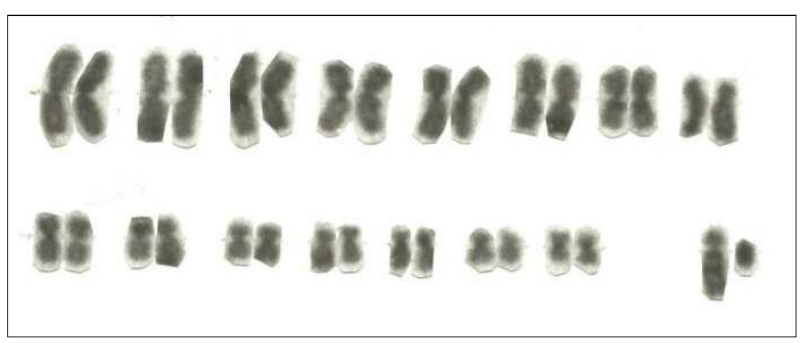

A

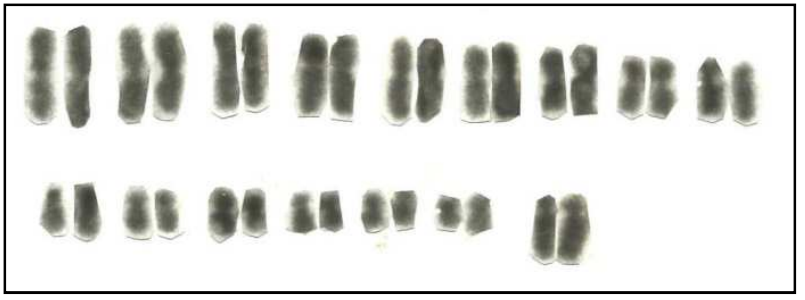

B

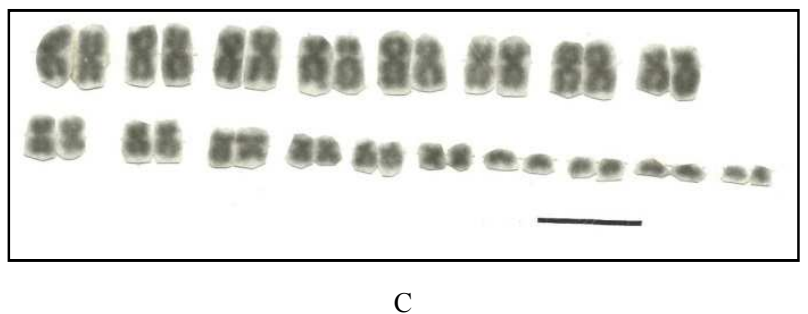

Figure 1. Karyotypes of A. Male Hipposideros caffer, B. Female Hipposideros caffer, C. Female Triaenops persicus. Bar $=2.4 \mu \mathrm{m}$.

\subsection{Family Mollosidae}

Three species in two genera of this family, Cherephon leucogaster, Cherephon pumila, and Mops condylura, were studied. The specimens were captured from Woliso, Batu and Arbaminch towns, respectively.

\subsubsection{Chaerephon Leucogaster}

In Figure $2 \mathrm{~A} \& \mathrm{~B}$ the karyotypes of male and female Chaerephon leucogaste, are presented and show $2 \mathrm{n}=48$ chromosomes. Four pairs of the autosomal chromosomes are metacentric and nineteen pairs are acrocentrics. The largest pair belongs to the metacentrics, the rest of the metacentrics being of medium size. Among the acrocentrics a few pairs appear to possess small second arm and may be acrocentric. In the male, the heteromorphic pair is consisting of a metacentric and a acrocentric chromosome. In the female karyotype, the corresponding pair is metacentric, indicating that $\mathrm{X}$ is metacentric and $\mathrm{Y}$ is acrocentric. The autosomal fundamental number is 54 , disregarding the few pairs which are apparently acrocentric chromosomes.

\subsubsection{Chaerephon Pumilus}

Figure 2C presents the karyotype of female Chaerephon pumilus, consisting of $2 \mathrm{n}=48$ chromosomes. The chromosome complement is consisting of five pairs of metacentric, eleven pairs of acrocentric-subacrocentric and eight pairs of acrocentric chromosomes. One pair of the 
medium sized metacentrics is likely to be the $\mathrm{X}$ chromosomes when compared to the karyotype of Chaerephon leucogaster, for which male and female karyotypes are available. Hence the fundamental number may be 54 , considering the rest are acrocentric.

\subsubsection{Mops Condylura}

As the female karyotype in Figure 2D shows, the $2 n=48$ chromosomes which are consisting of one pair of large and four pairs of medium sized metacentrics and nineteen pairs of acrocentrics, with a few apparently acrocentric looking pairs among the latter. The autosomal fundamental number is 54 , when the acrocentric looking pairs are disregarded. By comparing to the karyotype of Chaerephon leucogaster, $\mathrm{X}$ chromosome could be one of the medium sized metacentrics.

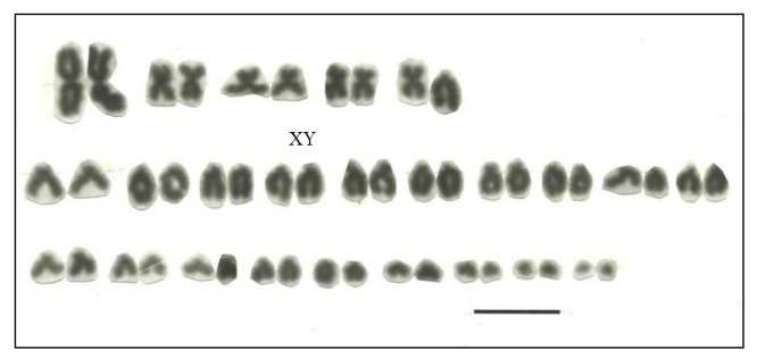

A

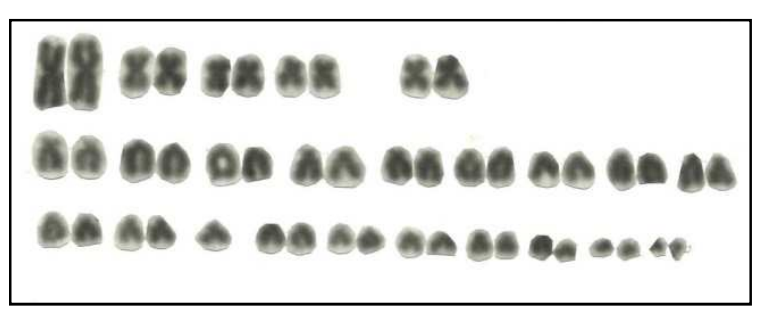

B

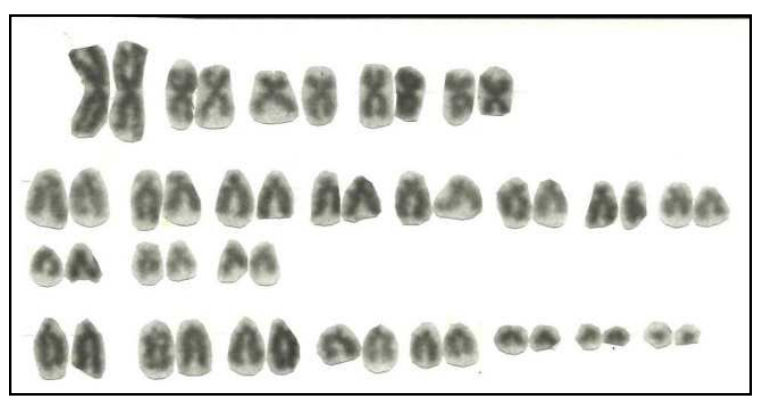

C

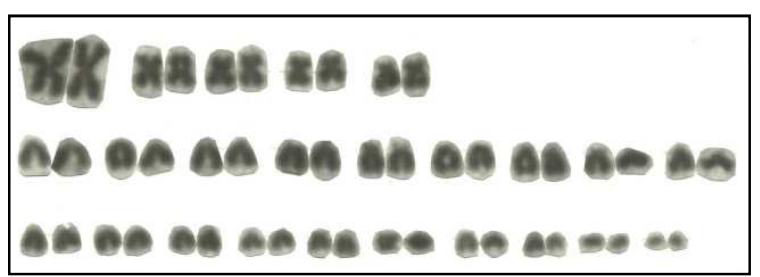

D

Figure 2. The karyotypes of three species of bats of the family Mollosidae. A. Male Chaerephon leucogaster; B. Female Chaerephon leucogaster, C. Female Chaerephon pumilus, D. Female Mops condylura.

\subsection{Family Nycteridae}

Only one species, Nycteris thebaica, was studied from this family. The specimen was captured from Dimdim Cave, in the vicinity of Alem Ketema, in the Merehabete district. The karyotype of a female specimen (Figure 3) shows $2 \mathrm{n}=42$ chromosomes. All chromosomes are bi-armed, except the smallest pair, which is acrocentric. Among the bi-armed chromosomes, those with two arms, all are metacentrics except three pairs (pairs 1, 11 and 12) which are submetacentrics. The sex chromosome could not be distinguished since a male karyotype is not available. The autosomal fundamental number could either be 80 if the acrocentric pair is the $\mathrm{X}$ chromosomes or 78 , if $\mathrm{X}$ is one of the bi-armed chromosomes.

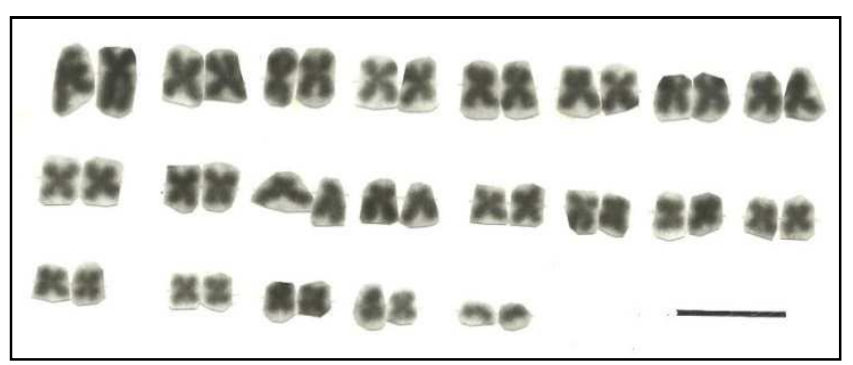

Figure 3. Karyotype of a female bat of Nycteris thebaica.

\subsection{Family Pteropodidae}

Only one species, Micropteropus pusillus, was studied from this family. It is also the only species from suborder Megachiroptera to be included in the present study. Specimens were collected from Koka, Batu and Arbaminch towns. Karyotypes of male and female are presented in Figure $4 \mathrm{~A} \& \mathrm{~B}$ and the female karyotype shows $2 \mathrm{n}=36$ chromosomes. In the female karyotype, all the chromosomes are bi-armed, with predominantly metacentrics and a few pairs of sub-metacentrics. In males, we were able to count only 35 chromosomes even in cells with well spread metaphase chromosomes. When the 35 chromosomes of males are arranged into putative homologous pairs on the basis of morphological similarity, a metacentric chromosome remains unpaired (Figure 4B). When compared to the female karyotype, the unpaired chromosome may be taken for $\mathrm{X}$ chromosome. The Y chromosome might be too small and escape cytological detection. It might be similar to the small chromatin material attached to another chromosome shown in Figure 4 (arrow), which we suspect it be the $Y$ chromosome. The autosomal fundamental number is 68 .

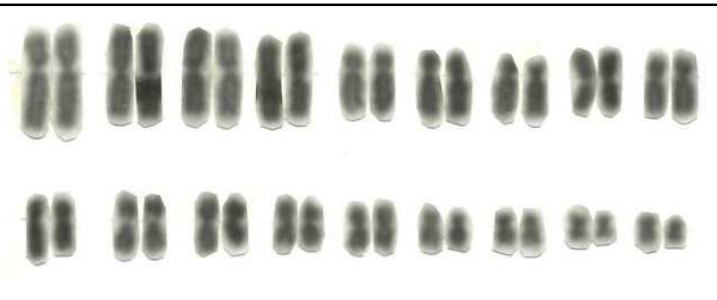

A. 


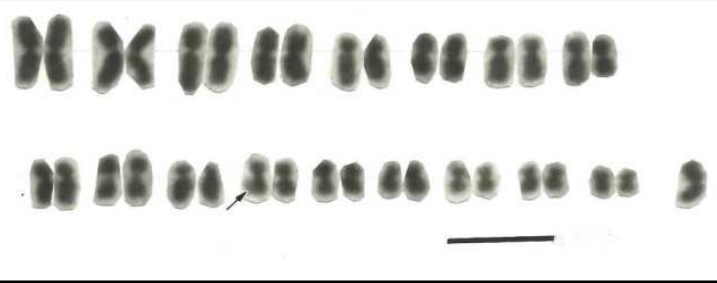

B.

Figure 4. Karyotype of Micropteropus pusillus. A. Female specimen from Koka town; B. male specimen from Batu town. Arrow indicates chromatin material attached to a chromsosme, suspected for Y chromosome.

\subsection{Family Vespertilionidae}

Four species from four genera of the family Vespertilionidae were studied. The studied species are Miniopterus africanus, Pipistrellus pipistrellus, Neoromicia nanus, and Scotophilus dingani/viridis, and their karyotypes are described below.

\subsubsection{Miniopterus Africanus}

A male specimen of this species was captured from a cave (Bolo) near Fiche town. The karyotype is presented in Figure $5 \mathrm{~A}$. The $2 \mathrm{n}$ chromosome number is 46 . The chromosomes are consisting of two large, two medium and one small pairs of metacentrics and seventeen pairs of acrocentrics, whose sizes descend gradually from medium to small. Although it is difficult to precisely determine the centromeric positions, the $\mathrm{X}$ chromosome appears a small metacentric and the $\mathrm{Y}$ a small dot-like acrocentric. The autosomal fundamental number is 54 .

\subsubsection{Pipistrellus Pipistrellus}

The specimens were captured from a banana plantation in Batu town. The karyotypes of female and male bats are presented (Figure 5B\&C). The $2 \mathrm{n}=36$ chromosomes are consisting of seven pairs of large metacentrics, one pair of small meta/sub-metacentrics and ten pairs of acrocentrics, the latter gradually descending in size. The male karyotype shows a heteromorphic pair of large and small acrocentric chromosomes, which, respectively, are $\mathrm{X}$ and $\mathrm{Y}$ chromosomes. Thus, in the female karyotype, the largest pair of acrocentrics must be $\mathrm{X}$ chromosome. The autosomal fundamental number for this species is 50 .

\subsubsection{Neoromicia Nanus}

Figure 5D presents the karyotype of female Neoromicia nanus, captured from a banana plantation in Arbaminch town. The $2 n=36$ chromosomes are consist of seven pairs of large metacentrics, a small sub-metacentric pair and ten pairs of acrocentrics. The karyotype is identical to that of the Pipistrellus pipistrellus described above. In line with the karyotype of Pipistrellus pipistrellus, the largest acrocentric in Neoromicia nanus must be $\mathrm{X}$ chromosome pair. The autosomal fundamental number of this species is 50 .

\subsubsection{Scotophilus Species}

A male specimen belonging to the genus Scotophilus was captured from Batu town. The karyotype showing $2 \mathrm{n}=36$ chromosomes is presented in Figure 5E. The autosomal chromosomes are consisting of three pairs of large, two pairs of medium and one pair of small metaphase; five pairs of acrocentric and six pairs of acrocentric chromosomes. A medium sized metacentric and a small acrocentric chromosomes form a heteromorphic pair, assumed to be $\mathrm{X}$ and $\mathrm{Y}$ chromosomes, though it difficult to distinguish between $\mathrm{X}$ and $\mathrm{Y}$ when male karyotype is not available. The autosomal fundamental number is 56. Regarding the taxonomy, it was not possible to establish the specimen either as Scotophilus dingani or Scotophilus viridis on the external morphology alone. The skeletal data that might help for taxonomic identification were not available.

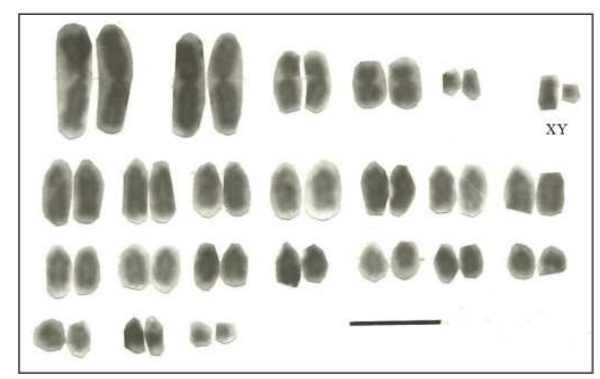

A.

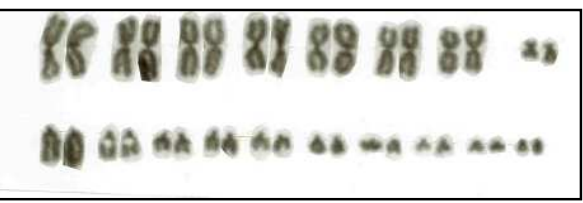

B.

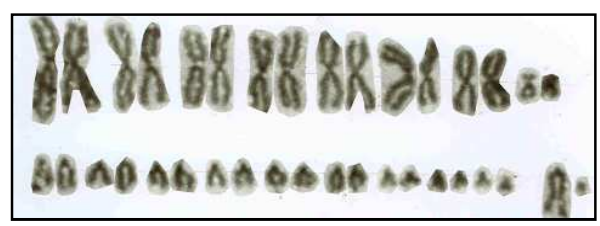

C.

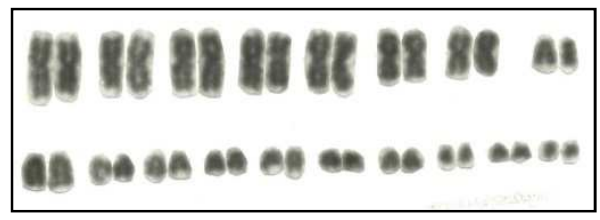

D.

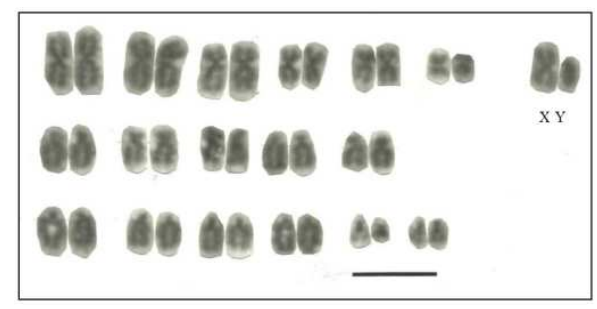

E.

Figure 5. Karyotypes of four species of bats of the family Vespertilionidae: A. Male Miniopterus africanus, B. Female Pipistrellus pipistrellus, C. Male Pipistrellus pipistrellus, D. Female Neoromicia nanus, E. Male Scotophilus spp. (dingani/viridis). Bar $=2.4 \mu \mathrm{m}$. 


\section{Discussion}

\subsection{Family Hipposidridae}

Family Hipposidridae comprises nine extant genera and about 80 species [22]. Hipposideros caffer and Trianops persicus captured from Bishoftu were included in the present study. Petersen and Negorsen ([20] reported $2 \mathrm{n}=$ 32 and autosomal fundamental number of 60 for Hipposideros caffer from Zimbabwe, which is in agreement with our present findings for Ethiopian specimens. They reported acrocentric $\mathrm{Y}$ chromosome and autosomal fundamental number of 60 , all in agreement with our present findings for the Ethiopian specimen of this species. However, Porter et al. [21] described the putative $\mathrm{Y}$ chromosome as bi-armed.

Trianops persicus, was proposed to be split into three species as a result of Cytochrome $b$ gene-based molecular analysis: namely T. afer in Africa, T. persicus and T. parvus sp. nov. in the Middle East [29]. According to Dulie and Mutere (as cited in [28] T. afer has $2 \mathrm{n}=36$ and $\mathrm{FN}=60$, medium sized metacentric $\mathrm{X}$ and small subtelocnetric $\mathrm{Y}$. This result much with our findings, however from our female specimen we couldn't exactly determine the FN as 60 . Hence, the species has a karyotype of 13 pairs of metacentrics and four pairs of acrocentric autosomal chromosomes and metacentric X/small Y subtelocentric.

\subsection{Family Molossidae}

This family comprises of two sub-families and 16 genera with about 100 species, of these about 40 species are known to occur in Africa. In its geographic distribution, the family is found in the warm tropics of both the Old World and New Worlds [24].

The chromosome number recorded for Molissidae family ranges $2 \mathrm{n}=34$ to 58 , but the most common karyotype is $2 \mathrm{n}=$ 48. The karyotype is usually characterized by the presence of one large metacentric and three medium-sized biarmed autosomal chromosomes.

The three species of this family, investigated in the present study, have the most common chromosome number of $2 \mathrm{n}=$ 48. These species, Chaerephon leucogaster, Chaerephon pumilus, and Mops condylura have very similar karyotypes. For example, the presence of the largest metacentric pair and three pairs of medium sized autosomal metacentrics, and a metacentric $\mathrm{X}$ chromosome are common features of the karyotypes of the three species. The $\mathrm{Y}$ chromosome of $C$. leucogaster is acrocentric. Not possible to know the morphology of Y chromosome in the other two species, since no male karyotypes were available for them.

Sreepada et al. [24] reported that in C. pumilus and $C$. plicatus from Senegal, the $\mathrm{X}$ chromosome is metacentric and $\mathrm{Y}$ is acrocentric, which is in agreement with the present study. However, in both species, they found $\mathrm{Y}$ is smaller than $\mathrm{X}$ whereas, in the present finding, $\mathrm{Y}$ and $\mathrm{X}$ are of about similar size, at least in C. leucogaster, it was possible to compare the two types of chromosomes.

\subsection{Family Nycteridae}

Nycteris thebaica (slit-faced bats) was the only species accessed from a cave, for this study. The diploid chromosomes, in this family, range from 34-to-42 [31], however, $2 \mathrm{n}=42$ seems conservative as reported similarly for other species of the genus Nycteris [32]; [33]; Porter et al. 2010). Peterson and Nagorsen [20] investigated $2 n=42$, and $\mathrm{FN}=78$ for $N$. thebaica, which fully matched with our result.

The karyotypes for this species as reported from Gabon showed 9 pairs of metacentric 7 sub-metacentric and 3 subacrocentric and one pair areocentric autosomes [21]. This report fits with our findings, except slight difference in classification of chromosomes, as sub-metacentric and subacrocentric and sex chromosomes which we couldn't identified from female specimen's karyotype. However, Porter et al. [21] reported sex chromosome as X chromosome metacentric and $\mathrm{Y}$ as small acrocentric.

\subsection{Family Pteropodidae}

Micropteropus pusillus (Peters' dwarf epauletted fruit bat), is usually smaller in size than its single sister species, Micropteropus intermedius, and distributed in many African countries such as Senegal, Sudan, Ethiopia, Kenya, Tanzania, Uganda, Congo, and Angola [7].

This family showed a wider range of diploid chromosome number (2n) 24 to 58 , but the three most common $2 \mathrm{n}$ so far reported are 34, 36, and 38 (Sotero-Caio et al. 2017).

Although not confirmed for this species, the mechanism of multiple sex chromosomes, which is common in phyllostomid bats may explain the variable chromosome number between sexes of our specimens, i.e., males $(2 n=35)$ and females $(2 n=36)$ or as it has been postulated in African pteropodids $\mathrm{Y}$ chromosome might underwent rearrangements [23] or it might be too small and escape cytological detection (or attached with other chromosomes). However, Haiduk et al. [6] claimed that the occurrence of two $\mathrm{Y}$ chromosomes in $M$. pusillus: one submetacentric and the other subacrocentric, while $2 \mathrm{n}=35$ and $\mathrm{FN}=64$, which means 16 bivalents metacentric autosomes, one $\mathrm{X}$ and two $\mathrm{Y}$ chromosomes in male, whereas in females 16 bivalent autosomes and four (X) chromosomes. The later investigation should be confirmed otherwise our proposal of tiny $\mathrm{Y}$ chromosome attached to one of autosomal chromosome, as indicated in Figure 4B, must be supported by molecular techniques.

Another explanation is that the autosomes may be 34 and two $(\mathrm{XX})$ in females and one (X0) in males like in the case of Epomops buettikoferi [30]; hence the fundamental number for M. pusillus is 68 rather than 64 .

\subsection{Family Vespertilionidae}

The species of this family (Vesper bats) are widely distributed, feed on insects, and successful group of bats. They consist of 43 genera and 343 species [13] [19]). In Ethiopia, 13 genera consisting of 27 species were recorded by Largen, et al. [15]. 
In this study, karyotypes of four bat species of this family were investigated. The specimens of Miniopterus africanus $(2 \mathrm{n}=46, \mathrm{FN}=54)$ were collected from Fiche area (Danisa Kebele), while roosting in cave. Previous reports to other species in this genus (M. schreibersii), support this finding in $2 \mathrm{n}$ and FN [2]. The karyotype for this species was two large, one medium and one small pair of metacentric and 18 pairs of acrocentric autosomes, which differs slightly from our $M$. africanus in two medium pairs of biarmed autosomes (not one pair) and smallest $\mathrm{Y}$ chromosome (rather than "the size of third smallest autosomes". Karatafi et al. [11] also reported $2 \mathrm{n}=46$ but variable $\mathrm{FN}(48,50$, and 52) for M. schreibersi.

The specimens of Pipistrellus pipistrellus and Neoromicia nanus were collected from inside of banana's pseudo-stem trunk, where they commonly roosting, from Batu and Arbaminch banana plantation, respectively. They have the same $2 \mathrm{n}=36$ and $\mathrm{FN}=50$. Their karyotypes look the same and cannot be distinguished from one another by the conventional methods of cytogenetics. It was previously reported that the diploid number of the genus Pipistrellus varies from $2 n=24$ to $2 n=46$, but the common $2 n$ is 44 [4]. For instance, Peterson and Nagorsen [20] reported 2n $=36$ and $\mathrm{FN}=50$ for $P$. nanus collected from Kenya and Rhodesia, which match with karyotype of $N$. nanus in this study. Other similar reports on karyotype $(2 \mathrm{n}=36$ and $\mathrm{FN}=$ 48) from the same genus are P. affinis (Patnak and Sharma 1969) and $P$. endoi [1], indicates they might be closely related to $N$. nanus.

The karyotype of Scotophilus dingani/viridis, $(2 \mathrm{n}=36 \mathrm{FN}$ = 56) we investigated agreed with the recent report from South Africa (for Scotophilus dingani) in diploid chromosome $(2 \mathrm{n}=36)$ but differs in fundamental number $(\mathrm{FN}=50)$ [3]. Eick et al. [3] claimed that the karyotype they discovered was by far different from other species of the sister species, reported previously, especially the fundamental number $(\mathrm{FN}=62)[20]$.

\section{Conclusion}

The bat systematic and evolutionary studies need karyotypic and molecular evidences, which further applied for ecological and conservational issues. Therefore, this current karyotypic study of 11 bat species is the first attempt towards cytogenetical and molecular data development of Ethiopian bats, part of the global conservation endeavor.

Hence, this study has provided, for the first time, karyotypic description of 11 species of bats from Ethiopia and compared the results with various related previous reports from other countries. This study has also contributed an additional bat species to the list of bats recorded from Ethiopia (Chaerephon leucogaster).

Each bat species is characterized by specific karyotypes. However, closely related species show related karyotypes than distantly related species. Representatives of the Molossidae family showed asymmetric karyotype (and one largest pair) while Hipposideridae groups seem symmetric type. The representative of Vespertilionidae family also showed asymmetric kind of karyotypes. The karyotypes of Scotophilus dingani and Neoromicia nanus represent somewhat bimodal karyotype. In Micropteropus pusillus and Nycteris thebaica all the chromosomes, except one pair in the later species, were bi-armed and decrease in size uniformly.

Diploid chromosome numbers are more reliable cytotaxonomic features than fundamental numbers, which show discrepancy from author to author for the same species. This can be ascribed to the fact that in subacrocentric type of chromosomes the second arm may sometimes escape detection. This would create difference in classifying chromosome as biarmed or uniarmed.

\section{Funding}

Addis Ababa University and Research Programme on Sustainable Use of Dryland Biodiversity (RPSUD)

\section{Availability of Data and Materials}

The authors declare that the data supporting the findings of this study are available within the article.

\section{Authors' Contributions}

Dr. Bekele and Mr. Mulugeta designed the experiment, performed the field specimen collection and laboratory work and analyzed the data and drafting the manuscript. Dr. Kifle involved in data interpretation editing the manuscript. All coauthors co-conceptualized the project, planned the experiment, revised the draft and approved the final manuscript.

\section{Acknowledgements}

Addis Ababa University, college of Natural and computational science and Research Programme on Sustainable Use of Dryland Biodiversity (RPSUD) funded this project.

\section{References}

[1] Ando K, Tagawa T, Uchida TA (1977) Considerations of karyotypic evolution within Vespertilionidae. Experientia, 33877-979.

[2] Bickham JWI, Hafner JC (1978) A chromosomal banding study of three species of vespertilionid bats from Yugoslavia. Genetica, 48 (1): 1-3.

[3] Eick GN, Jacobs DS Yang F, Volleth M (2007) Karyotypic differences in two sibling species of Scotophilusfrom South Africa (Vespertilionidae, Chiroptera, Mammalia). Cytogenet Genome Res 118: 72-77.

[4] Fedyk S, Ruprecht AL (1976) Karyotypes of Pipistrellus Pipistrellus (Schreber 1774) and P. nathusii (Keyserling and Blasius 1839) (Chiroptera: Vespertilionidae), Caryologia, 29 (3): 283-289. 
[5] Fredga K (1977) Chromosomal changes in vertebrate evolution. In: Harrison RJ (ed) Zoological Science. The Royal Society, London, pp 377-397.

[6] Haiduk MW, Baker RJ, Robbins LW, Schlitter DA (1981) Chromosomal evolution in African megachiroptera: G- and Cband assessment of the magnitude of change in similar standard karyotypes. Cytogenet Cell Genet; 29: 221-232.

[7] Hayman RW, Hill JE (1971) Order Chiroptera. In: Meester J, Setzer HW (eds) The mammals of Africa: an identification manual. Smithsonian Institution Press, Washington, District of Columbia, pp 1-73.

[8] Hill JE, Smith JD (1983) Bats: a Natural History. Natural History Museum, London University of Texas Press. 243.

[9] Hillis DM, Moritz C, Marble BK (1996) Molecular Systematics. $2^{\text {nd }}$ ed. Sinauer Associates, Inc. Publishers, Sunderland, Massachusetts, U.S.A. pp 121-168.

[10] Jepsen GL (1970) Bat origins and evolution. In: Wimsatt WA (ed) Biology of bats, vol. 1. New York: Academic Press, 1-64.

[11] Karatafi A, Gharakheloo MM, Kankilic T (2008) Karyotypes of Two Iranian Bat Species, Myotis blythii and Miniopterus schreibersii (Chiroptera: Vespertilionidae, Miniopteridae). Turk J Zool 32: 305-308.

[12] Kingdon J (1974) East African Mammals: Insectvores and bats. An Atlas of Evolution in Africa, Vol. 2 (A). Academic Press, London and New York.

[13] Koopman KF (1970) Zoogeography of bats. In: Slaughter BH, Walton DW (eds) About bats: a chiropteran biology symposium. Southern Methodist University Press. Dallas, Texas, pp 29-50.

[14] Kruskop SV, Lavrenchenko LA (2000) A new species of longeared bat (Plecotus; Vespertilionidae, Mammalia) from Ethiopia. Mammalia 38: 5-17.

[15] Largen MJ, Kock D, Yalden DW (1974) Catalogue of the Mammals of Ethiopia. J. Ital. Zool. 16: 221-298.

[16] Levan A, Fredga K, Sandberg AV (1964) Nomenclature for centromeric position on chromosomes. Hereditas 52: 201-220.

[17] Mitchell-Jones T (2003) Focus on bats discovering their lifestyle and habitats. Engl. Nature 2: 3-19.

[18] Nowak RM (1994) Walker's Bats of the World. The Johns Hopkins University Press, London.

[19] Nowak RM (1999) Walker's Mammals of the World, $6^{\text {th }}$ edn. Vol. 1. The Johns Hopkins University Press, Baltimore and Maryland.

[20] Peterson RL, Nagorsen DW (1975) Chromosomes of. fifteen species of bats (Chiroptera) from Kenya and Rhodesia. Life Science Occasional Papers - Royal Ontario Museum 27: 1-14.
[21] Porter CA, Primus AW, Hoffmann FG, Baker RJ (2010). Karyology of five species of bats (Vespertilionidae, Hipposideridae, and Nycteridae) from Gabon with comments on the taxonomy of Glauconycteris. Occasional Papers of the Museum, Texas Tech University 295: 1-7.

[22] Simmons NB (2005) Order Chiroptera. In: Wilson DE, Reeder DM (eds) Mammal species of the world: A taxonomic and geographic reference. The Johns Hopkins University Press Baltimore. pp 312-529.

[23] Sotero-Caio C, Baker RJ Volleth M (2017) Chromosomal Evolution in Chiroptera. Genes, 8 (272): 1-25; http//doi:10.3390/genes8100272

[24] Sreepada KS, Koubínova D, Konecny A, Koubek P, Rab P, Rabova MV, Zima J (2008) Karyotypes of three species of molossid bats (Molossidae, Chiroptera) from India and western Africa Folia Zool. 57 (4): 347-357.

[25] Williams-Whitmer LM, Brittingham-Brant M (2001) Wildlife damage control 4: Bats (http://www.cas.psu.edu).

[26] Wilson ED, Cole RF, Nichols DJ, Rudran R, Foster SM (1996) Measuring and Monitoring Biological Diversity: Standard Methods for Mammals. Smithsonian Institution Press, Washington and London.

[27] Yalden DW, Morris PA (1975) The Lives of Bats. David and Charles Limited, London.

[28] Happold M, Cotterill FPD (2013) Family rhinolophidaehorseshoe bats. In: Kingdon J, Happold D, Hoffmann m BT, Happold M, Kalina, J (eds) Mammals of Africa, vol. 1. Bloomsbury publishing, London, New Delhi, New York and Sydney, pp 300-356.

[29] Petr B, Peter V (2009) Taxonomic revision of the genus Triaenops (chiroptera: hipposideridae) with description of a new species from southern arabia and definitions of a new genus and tribe. Folia Zoologica; Praha 58: 1-45.

[30] Harada M, Minezawa M, Takada S, Yenbutra S, Nunpakdee P, Ohtani S (1982) Karyological analysis of 12 species of Bats from Thailand. Caryologia 35 (2): 269-278.

[31] Denys C, Kadjo B, Missoup AD, Monadjem A, Aniskine V (2013) New records of bats (Mammalia: Chiroptera) and karyotypes from Guinean Mount Nimba (West Africa), Italian Journal of Zoology, 80: 2, 279-290.

[32] Rautenbach I. L, Bronner GN, Schlitter DA (1993) Karyotypic data and attendant systematic implications for the bats of southern Africa. Koedoe 36 (2): 87-104.

[33] Lee TE, Bickham JW, Schlitter DA (1989) Karyotypes of two nycterid bats from Somalia. Mammalia 53: 120-121. 